\title{
Intubation naso-trachéale versus intubation oro-trachéale en chirurgie orale : étude rétrospective sur 809 patients au CHR de Metz.
}

\author{
Blein E1, Bridonneau T², Profumo L², Schwenck D¹, Bahi Gross S², Gérard E1 \\ 1. CHR Metz Thionville \\ 2. Les Hôpitaux Universitaires de Strasbourg (HUS)
}

L'anesthésie générale pour les actes de chirurgie orale est une alternative lorsque ceux-ci ne peuvent être réalisés sous anesthésie locale. En chirurgie orale, elle a une place spécifique et originale. II s'agit d'une intervention de l'étage céphalique : chirurgiens et anesthésistes doivent se partager un espace commun, le carrefour aérodigestif en préservant la liberté des voies aériennes. Il existe plusieurs types de gestions des voies aériennes en chirurgie orale : l'intubation oro-trachéale et l'intubation naso-trachéale sont les plus courantes. Cette dernière peut provoquer de nombreuses complications : incident mineur (épistaxis) mais aussi sévères (perforation septale, abcès du septum, abrasion turbinale, brèches de la lame criblée) et entraîner diverses séquelles (synéchies, obstructions nasales, sifflement nasal, sinusites chroniques).

Cette étude monocentrique rétrospective concerne 809 patients ayant bénéficié d'une intervention de chirurgie orale au bloc opératoire sous anesthésie générale au CHR de Metz-Thionville. Les chirurgies ont été regroupées en 5 catégories :

- dents de sagesse

- drainage de cellulite avec avulsion de la dent causale

- extractions dentaires multiples (E.D.M.)

- soins pédodontiques

- autres chirurgies : dents incluses (avulsion ou dégagement chirurgical pour traction orthodontique, implants, exérèse de kyste, chirurgie des tissus mous). L'objectif principal était les complications lors de l'intubation naso-trachéale. Elles étaient faibles : de l'ordre de 0,3\%. Dans cette étude, seules les épistaxis persistantes après le passage du patient en Salle de Surveillance Post-Interventionnelle ont été relevées. L'objectif secondaire de cette étude était la mise en évidence d'une différence des complications post-opératoires lors des intubations trachéales. $p=0,11$, aucune différence n'a pu être mise en évidence.

II n'existe pas de recommandations pour la gestion des voies aériennes lors d'une anesthésie générale en chirurgie orale. Si le choix du type d'intubation reste la décision du médecin anesthésiste, le chirurgien préfère l'intubation naso-tachéale car elle permet un libre accès à la cavité buccale. Compte tenu de l'absence de différence de complications mise en évidence lors de cette étude entre les 2 techniques, elle pourrait être considérée comme la technique de choix lorsqu'elle est possible dans ce type de chirurgie.

eleonoreblein@gmail.com

(C) The authors, published by EDP Sciences. This is an Open Access article distributed under the terms of the Creative Commons Attribution License 4.0 (http://creativecommons.org/licenses/by/4.0/) 